\title{
Hysteretic control of organic conductance due to remanent magnetic fringe fields
}

Cite as: Appl. Phys. Lett. 102, 042408 (2013); https://doi.org/10.1063/1.4790141

Submitted: 29 November 2012 . Accepted: 18 January 2013 . Published Online: 01 February 2013

F. Macià, F. Wang, N. J. Harmon, M. Wohlgenannt, A. D. Kent, and M. E. Flatté
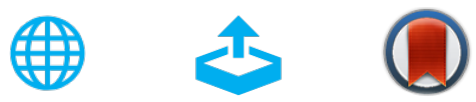

View Online

Export Citation

\section{ARTICLES YOU MAY BE INTERESTED IN}

Manipulation of the electroluminescence of organic light-emitting diodes via fringe fields from patterned magnetic domains

Applied Physics Letters 109, 243303 (2016); https://doi.org/10.1063/1.4971332

A two-site bipolaron model for organic magnetoresistance

Journal of Applied Physics 103, 07F303 (2008); https://doi.org/10.1063/1.2828706

Conversion of spin current into charge current at room temperature: Inverse spin-Hall effect

Applied Physics Letters 88, 182509 (2006); https://doi.org/10.1063/1.2199473

Lock-in Amplifiers Find out more today

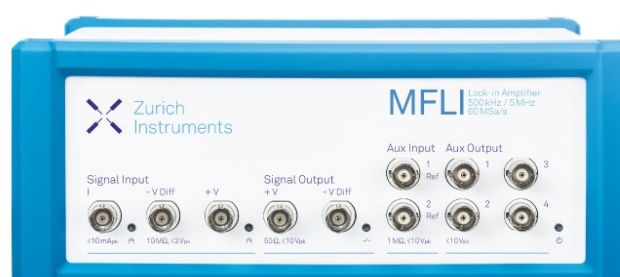

Zurich Instruments 


\title{
Hysteretic control of organic conductance due to remanent magnetic fringe fields
}

\author{
F. Macià, ${ }^{1}$ F. Wang, ${ }^{2}$ N. J. Harmon, ${ }^{2}$ M. Wohlgenannt, ${ }^{2}$ A. D. Kent, ${ }^{1}$ and M. E. Flatté ${ }^{2}$ \\ ${ }^{1}$ Department of Physics, New York University, 4 Washington Place, New York, New York 10003, USA \\ ${ }^{2}$ Department of Physics and Astronomy and Optical Science and Technology Center, University of Iowa, \\ Iowa City, Iowa 52242, USA
}

(Received 29 November 2012; accepted 18 January 2013; published online 1 February 2013)

\begin{abstract}
Manipulation of the remanent (zero external magnetic field) magnetization state of a single ferromagnetic film is shown to control the room-temperature conductance of an organic semiconductor thin film deposited on top. For the organic semiconductor $\mathrm{Alq}_{3}$, the magnetic fringe fields from a multidomain remanent magnetization state of the film enhance the device conductance by several percent relative to its value for the magnetically saturated ferromagnetic film. The effect of fringe fields is insensitive to ferromagnetic film's thickness (which varies the fringe field magnitude proportionately) but sensitive to the magnetic domain's correlation length. (C) 2013 American Institute of Physics. [http://dx.doi.org/10.1063/1.4790141]
\end{abstract}

Magnetoconductivity in organic thin film devices has been the subject of extensive study, particularly with the discovery that very small external magnetic fields $(\sim 10 \mathrm{mT})$ can lead to changes in conductance of several percentage at room temperature, ${ }^{1-7}$ an effect known as "organic magnetoresistance" or OMAR. Several mechanisms for this effect have been proposed, ${ }^{3,6-8}$ including electronic transport in spatially varying and randomly oriented molecular hyperfine fields. ${ }^{6}$ The mechanism for affecting electronic transport through such inhomogeneous random fields suggests that the fringe fields from a ferromagnetic film might similarly modify electronic transport, whether or not the OMAR mechanism originates from hyperfine interactions. Recently this effect, called "fringe-field OMAR," was identified in measurements where the fringe fields from a single unsaturated ferromagnetic film dramatically influenced an organic film's conductance. ${ }^{9}$ The conductance was found to be suppressed by several percent at room temperature near the coercive field of the magnetic film, when domains form as the ferromagnet's magnetization reverses. The effect was found to originate from the ferromagnet's spatially varying fringe fields, which leads to a spatially varying electron spinquantization axis and suppresses the device conductance (similar to mechanisms for hyperfine field-induced magnetoresistance). Erasing the domain structure with an applied field much larger than the anisotropy field enhances the device conductance. We note that much smaller effects on the electroluminescence in organic spin-valve devices were observed earlier by Salis et al. ${ }^{10}$ and assigned to effects from the uniform fringe field of a saturated magnetic layer.

Here, we characterize the effect of remanent fringe fields from a ferromagnetic film on an organic's conductance. At zero applied field, these remanent fringe fields increase the conductance of the organic semiconductor tris(8-hydroxyquinolinato)aluminium $\left(\mathrm{Alq}_{3}\right)$, suppressing $\mathrm{OMAR}$, in contrast to fringe fields from a similar domain configuration generated at the coercive field (where the film magnetization begins to switch) which decrease the $\mathrm{Alq}_{3}$ conductance, as we reported in Ref. 9. We also demonstrate that the thickness of the ferromagnetic film (and thus the proportionally increasing fringe field magnitudes) is not critical to the observed effect and can be varied by a factor of four without significantly changing the magnitude of the fringe field OMAR effect. Since one expects that the domain size increases with layer thickness (see also Fig. 1), these results appear to be consistent with the gradient of the fringe fields being the most important factor influencing the magnetic-field-dependence of the electronic transport in the organic layer.

Our devices consist of an organic thin film diode fabricated on top of a ferromagnetic thin film. The thin film diode employs poly (3,4-ethylenedioxythiophene)-poly (styrenesulfonate) (PEDOT) as its non-magnetic bottom electrode and $\mathrm{Ca}$ as its top electrode (see schematic in Fig. 1(a)). PEDOT and $\mathrm{Ca}$ are commonly used electrodes for organic devices such as light-emitting diodes. The ferromagnetic thin film has perpendicular anisotropy and forms small scale $(\sim 500 \mathrm{~nm})$ domains near the film's coercive field (Fig. 1(b)). There are strong fringe fields emanating from the ferromagnetic film when it is in a multi-domain state. The ferromagnetic layer consists of a cobalt (Co) and platinum (Pt) multilayer, $[\mathrm{Co} \mid \mathrm{Pt}] \times n$, where $n$ is the number of bilayer repeats and sets the thickness of the layer $t$. The number of bilayer repeats has been varied from $n=5(t \sim 4 \mathrm{~nm})$ to $n=22(t \sim 18 \mathrm{~nm})$ to change the magnitude of the fringe fields. The thin films we have used for this study have $n=5,10,15$, and 22 .

Magnetization reversal in the ferromagnetic film occurs through nucleation, growth, and annihilation of magnetic domains. ${ }^{9,11}$ The strength of magnetic fringe fields induced by domains on a ferromagnet depends strongly (almost linearly) on $n$ or equivalently the thickness $t$ of the ferromagnetic layer. The upper panel of Fig. 1(b) shows the root mean square (rms) value of fringe fields $12 \mathrm{~nm}$ above the surface of a one dimensional periodic (up magnetized/down magnetized) domain structure with a $500 \mathrm{~nm}$ period as a function of the ferromagnet's thickness. The properties of $\mathrm{Co} \mid \mathrm{Pt}$ ferromagnetic films have been characterized in detail (see Ref. 9).

In previous work, ${ }^{9}$ we showed that the conductivity of an organic semiconductor on top of a ferromagnetic film was suppressed when the ferromagnet is in a multi-domain state. Figure 1(c) shows magnetoconductivity results for an $\mathrm{Alq}_{3}$ 
(a)

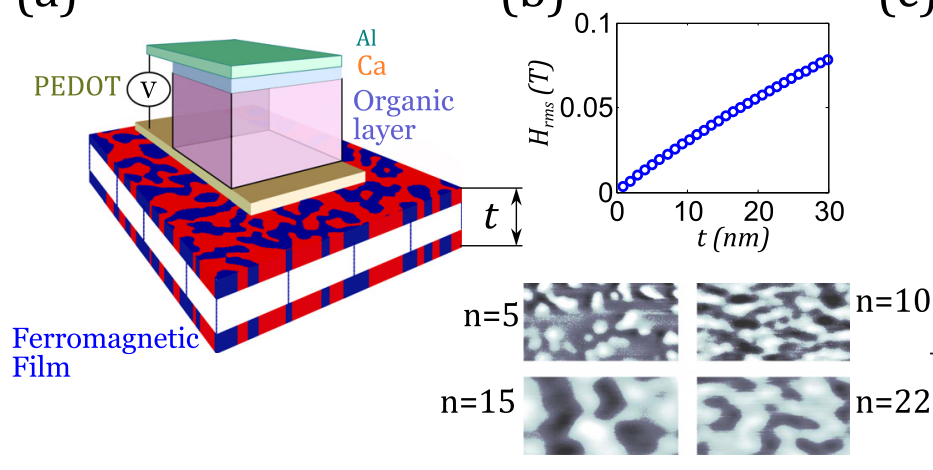

(c)

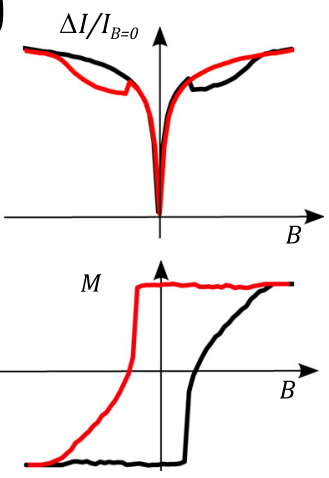

FIG. 1. (a) Schematic of the device, consisting of an organic layer on top of a ferromagnetic thin film. The thickness of the magnetic film, $t$, is varied by varying $n$, the number of Co|Pt bilayer repeats. (b) The rms value of the fringe fields $12 \mathrm{~nm}$ above the film surface for a $1 \mathrm{D}$ periodic domain structure, magnetized up/down with a $500 \mathrm{~nm}$ period, as a function of the film thickness. The lower panel shows MFM images of magnetic films with $n=5$, 10 , 15 , and 22 . Images

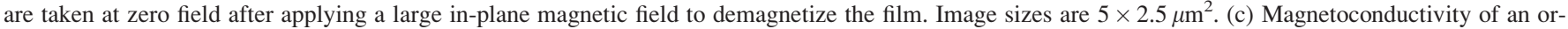
ganic thin film ( $\mathrm{Alq}_{3}$ on top of a $n=22$ ferromagnetic layer) and magnetization hysteresis loop with perpendicular applied field for the same device.

layer on top of a $n=22 \mathrm{Co} \mid \mathrm{Pt}$ layer along with the magnetization curve of the same ferromagnetic layer. We observe 3 conductance states. At zero applied field, the film is uniformly magnetized, there are no fringe fields, and only hyperfine fields are present in the organic layer; the conductivity is at a minimum. At large applied fields, the film is again uniformly magnetized; the conductivity is maximum and nearly independent of the applied field. In a field range around the coercive field of the magnetic film, the film is in a multi-domain state and produces fringe fields in the organic layer; the conductivity is suppressed. Note that this hysteretic fringe-field OMAR response occurs on top of the nonhysteretic, Lorentzian-like hyperfine OMAR traces.

In Fig. 2, we compare the magnetoconductivity of a $40 \mathrm{~nm}$ thick $\mathrm{Alq}_{3}$ film on top of different magnetic films (i.e., films with different number of bilayer repeats). The magnetic field is applied perpendicular to the film plane and swept smoothly from large negative to large positive (black lines) and back (red lines). Magnetic hysteresis loops are also plotted in the same graphs to show when the ferromagnet is in a multi-domain state and when it is saturated. There is a strong correlation between the regions where the magnetization is changing and the "dips" in the magnetoconductivity curves. Notice that the sample with $n=5$ has a sharp jump of magnetization-the sample goes from negative to positive magnetization without having intermediate states - and presents no features in the conductivity curve.

Magnetoconductivity in the same devices has been measured by sweeping the applied field in the film plane (Fig. 3). In this case, the magnetic film has almost no hysteresis and it saturates at fields larger than its anisotropy field $\left(B_{a} \simeq 1 \mathrm{~T}\right)$. At smaller fields, the ferromagnetic films are in a multi-domain state (i.e., equivalent to the states near to the coercive fields for out-of plane magnetic fields). The curves
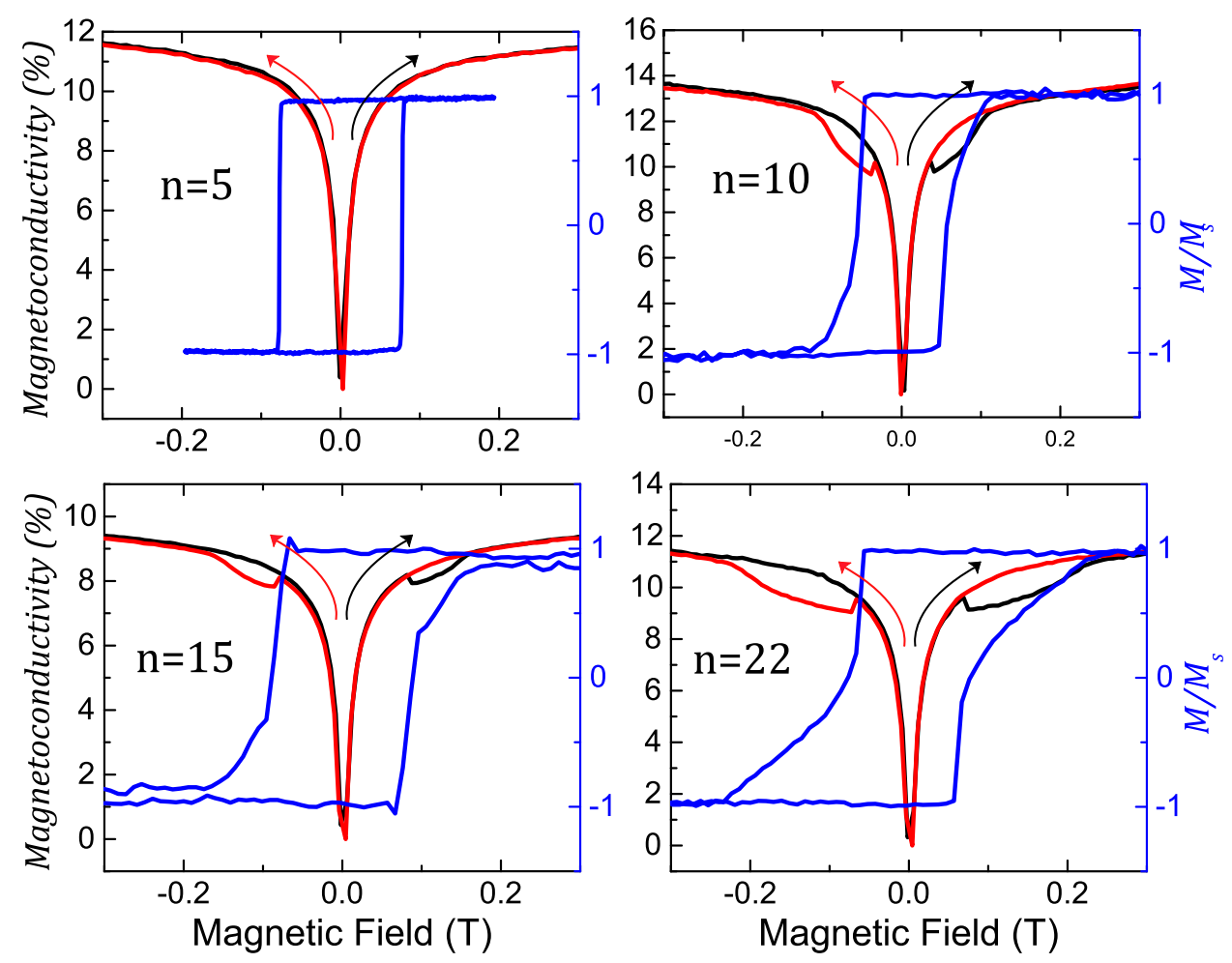

FIG. 2. Magnetoconductivity of devices with $25 \mathrm{~nm}$ of PEDOT and $40 \mathrm{~nm}$ of $\mathrm{Alq}_{3}$ on top of ferromagnetic thin films and magnetic hysteresis curves of the same films (applied field perpendicular to the film plane). Different panels correspond to different films with different coercive fields and thicknesses. 

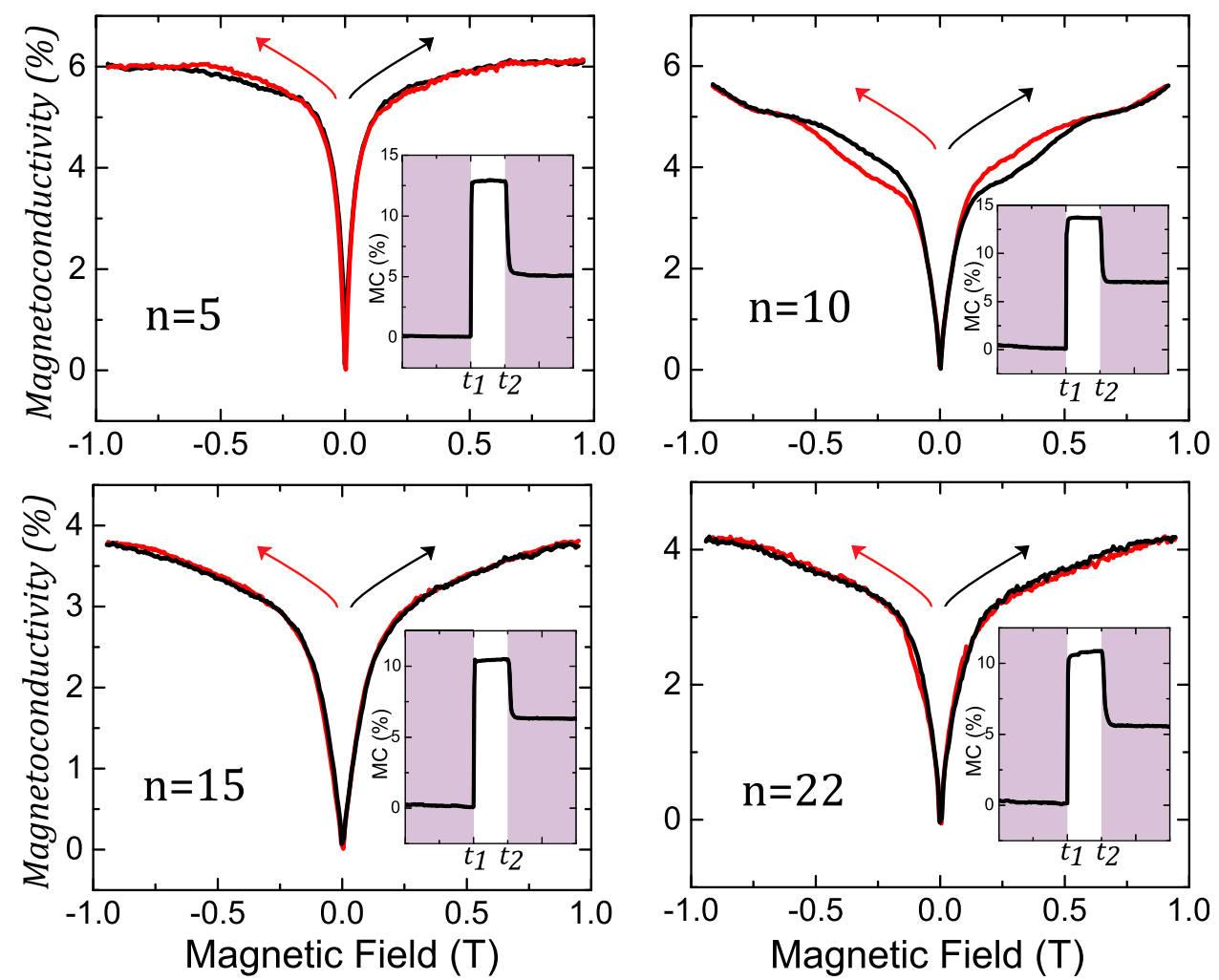

FIG. 3. Magnetoconductivity of devices with $25 \mathrm{~nm}$ of PEDOT and $40 \mathrm{~nm}$ of $\mathrm{Alq}_{3}$ on top of magnetic films (applied field in the film plane). Different panels correspond to different films with different thicknesses. Insets show time traces of magnetoconductivity in the same devices for a sequence of in-plane magnetic field from $0 \mathrm{~T}$ to $1 \mathrm{~T}$ and back to $0 \mathrm{~T}$; shaded areas correspond to zero applied field. In the initial state the ferromagnetic film was magnetized to its saturation value. shown in Fig. 3 resemble the curves in Fig. 2 with a difference, the "dip" characteristics are not visible but there is still a minimum conductivity at zero applied field (when the ferromagnetic layer is demagnetized). A closer look reveals that the magnetoconductivity at large fields is only $5 \%-7 \%$ while for the applied field perpendicular to the film, the magnetoconductivity was $9 \%-14 \%$. We would expect the conductivity at large fields to be equal in both Figs. 2 and 3 because saturated films do not produce any fringe field. However, the conductivity measured at zero applied field may be different between the two cases because in Fig. 2 the film is magnetically saturated at zero field, whereas in Fig. 3 the film has magnetic domains (i.e., has been demagnetized by applying this large in-plane field).

Our magnetic films can switch from magnetically saturated (single-domain) to magnetically unsaturated (multidomain) at zero applied field by applying a magnetic pulse (larger than $0.6 \mathrm{~T}$ ) in-plane to achieve a multi-domain state. We then designed a time and field sequence to systematically vary the ferromagnet's state at zero applied field from saturated to unsaturated state. We started with a fully magnetized layer at zero time; a $1 \mathrm{~T}$ in-plane magnetic field was applied at $t_{1}$ and later on, at $t_{2}$, removed, achieving a magnetically unsaturated state equivalent to the state measured at zero field for the curves in Fig. 3. Time traces for the described field sequences are plotted as insets in Fig. 3 for the four different ferromagnetic films. We see that the organic layer's conductivity varies by up to $7 \%$ when the state of the ferromagnet is changed; it is increased when the ferromagnet is in a multi-domain state. It is also remarkable that such conductivity changes are in the same range $(5 \%-7 \%$ ) for all four films (from $n=5$ to $n=22$ ).

The thinner ferromagnetic layer with $n=5$ presented a sharp magnetization switching and consequently no features in the conductivity curve in Fig. 2. However, the time- sequence insets in Fig. 3 show that a significant change in conductivity occurs when switching from a saturated to an unsaturated state configuration of this ferromagnetic layer.

To understand better these results-the different conductivity states in the absence of applied field seen in time traces in Fig. 3-we created specific remanent magnetization states in the ferromagnetic film with different domain configurations and measured the conductivity of the $\mathrm{Alq}_{3}$ layer. Different remanent states were achieved by applying a perpendicular field close to the film's coercive field and removing that field (see Fig. 4). We found through magnetization measurements that the remanent magnetization is approximately equal to the magnetization in this perpendicular field $\left(M_{r}=M(H)\right.$, $\left.H \sim H_{c}\right)$. In this way, the remanant magnetization could be varied from negative to positive saturation $\left(-M_{s}<M_{r}<M_{s}\right)$ and with it the magnetic domain configuration in zero field.

Figure 4 shows the conductivity of a $28 \mathrm{~nm}$ thick $\mathrm{Alq}_{3}$ film on top of a magnetic layer for the different remanent states. Positive magnetic field values set the ferromagnetic layer's magnetic state (blue dots) and the same magnetic state is approximately maintained at zero applied field (red dots). The measuring sequence is sketched in the upper panels of Fig. 4. We first saturated the sample with a large negative field, then we set a positive field value from 0 to $0.3 \mathrm{~T}$ and measured the conductivity of the organic layer (blue points), and then we removed the applied field and again measured the organic's conductivity (red points). The blue line reproduces the magnetoconductivity shown in Fig. 2, whereas the red line corresponds to the conductivity in the absence of applied field. Remanent-state curves in Fig. 4 show a first step that brings the conductivity to a higher value and corresponds to domain nucleation in the ferromagnet. Next, the conductivity remains almost constant while domains grow and then it drops back to the lower value 


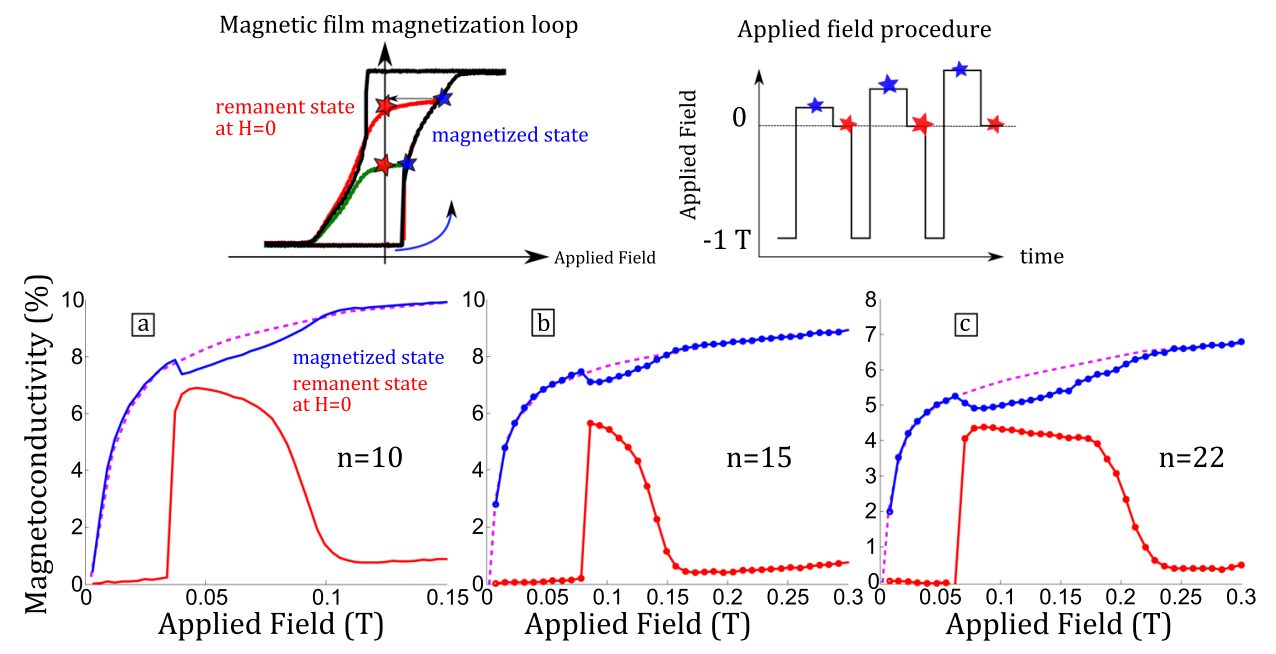

FIG. 4. Upper panel shows the field sequence to prepare remanent states at zero magnetic field: a large negative magnetic field saturates the ferromagnet, then a small positive field sets a magnetization state in the ferromagnet, and finally the field is reduced to zero and the ferromagnet remains at a remanent magnetization state. Panels (a)-(c) show results of the organic's conductivity for magnetic films of number of repeats $n$ and different fringe-field strengths. Conductivity curves are measured at values from the field sequence marked with stars in the schematic field sequence: positive applied field (blue) and at zero applied field (red). when domains annihilate. It is clear from these results that the zero field conductance is enhanced when the ferromagnetic film is in a multi-domain state. We also find that the remanent magnetization conductivity of $\mathrm{Alq}_{3}$ is nearly constant for electrodes magnetization values smaller than half of the saturation magnetization, $-0.5 M_{s}<M_{r}<0.5 M_{s}$.

The magnetic fringe fields provide an inhomogeneous local field landscape in addition to the nuclear hyperfine fields. Fringe fields suppress conductivity in $\mathrm{Alq}_{3}$ in the presence of a uniform applied field (see Fig. 4 blue points) and enhance the conductivity at zero applied field (red points). The conductivity value for a given fringe field configuration hardly varies with the uniform applied field, suggesting that the conductivity is entirely controlled by the fringe field configuration. Fringe fields from our magnetic films overwhelm hyperfine fields and at the same time are not overwhelmed by a uniform applied field of the order of the film's coercivity. We have calculated the fringe fields in the organic layer due to the magnetic films. The magnitude of the fringe fields are of the order of film's coercive field $(\sim 100 \mathrm{mT})$ and thus largely exceed the hyperfine fields. (Fig. 1(b) shows a calculation of the rms fringe field values.)

An interesting observation is that the effects appear to be independent of the ferromagnet film's thickness. This suggests that the strength of the fringe fields is not important as long as they are much larger than the hyperfine fields. Domain sizes of the ferromagnetic film appear to grow with film thickness; although fringe field magnitudes increase with ferromagnetic film thickness, their gradients may remain roughly constant if the domains increase in size correspondingly with increasing film thickness. Qualitatively, increasing a ferromagnet's thickness increases the magnetic anisotropy energy and decreases the importance of magnetostatic energy with respect to the exchange energy, leading to larger domain sizes. ${ }^{12}$ There are other parameters that may effect the competition between magnetostatic and exchange energies and therefore domain sizes. We do not have enough data to fully describe the domain sizes as a function of the ferromagnet film thickness. However, we observe a trend in Magnetic Force Microscopy (MFM) images at zero applied field (Fig. 1) of increasing domain size with increasing layer thickness. A theory that accounts for the field gradients that result from the combination of the fringe, applied and hyperfine fields, rather than only the field magnitudes, is being explored. ${ }^{13}$
Similar experiments have been conducted on poly [2-methoxy-5-(2-ethylhexyloxy)-1,4-phenylene-vinylene] (MEHPPV) as an organic semiconductor and similar results have been obtained. This indicates that this effect occurs both in materials whose non-fringe-field OMAR is known to occur via hyperfine fields (MEHPPV) and those where the mechanism for non-fringe-field OMAR is controversial, such as in $\mathrm{Alq}_{3}$ where spin-orbit interactions may be important. ${ }^{14}$

We have demonstrated sensitive control of the roomtemperature conductivity of an organic film via manipulation of the remanent state of a ferromagnetic film. The independence of this effect from ferromagnetic film thickness strongly suggests that fringe field gradients provide the mechanism for conductivity change.

F.M. acknowledges support from a Marie Curie IOF 253214. This work was supported by ARO MURI Grant No. W911NF-08-1-0317 and NSF Grant No. ECS 07-25280. We acknowledge assistance from $\mathrm{S}$. Shen and fruitful discussions with P. A. Bobbert.

${ }^{1}$ T. L. Francis, O. Mermer, G. Veeraraghavan, and M. Wohlgenannt, New J. Phys. 6, 185 (2004).

${ }^{2}$ V. Prigodin, J. Bergeson, D. Lincoln, and A. Epstein, Synth. Met. 156, 757 (2006).

${ }^{3}$ P. Desai, P. Shakya, T. Kreouzis, and W. P. Gillin, Phys. Rev. B 76, 235202 (2007).

${ }^{4}$ B. Hu and Y. Wu, Nature Mater. 6, 985 (2007).

${ }^{5}$ F. L. Bloom, W. Wagemans, M. Kemerink, and B. Koopmans, Phys. Rev. Lett. 99, 257201 (2007).

${ }^{6}$ P. A. Bobbert, T. D. Nguyen, F. W. A. van Oost, B. Koopmans, and M. Wohlgenannt, Phys. Rev. Lett. 99, 216801 (2007).

${ }^{7}$ J. D. Bergeson, V. N. Prigodin, D. M. Lincoln, and A. J. Epstein, Phys. Rev. Lett. 100, 067201 (2008).

${ }^{8}$ J. Kalinowski, M. Cocchi, D. Virgili, P. Di Marco, and V. Fattori, Chem. Phys. Lett. 380, 710 (2003).

${ }^{9}$ F. Wang, F. Macià, M. Wohlgenannt, A. D. Kent, and M. E. Flatté, Phys. Rev. X 2, 021013 (2012).

${ }^{10}$ G. Salis, S. F. Alvarado, M. Tschudy, T. Brunschwiler, and R. Allenspach, Phys. Rev. B 70, 085203 (2004).

${ }^{11}$ F. Macià, P. Warnicke, D. Bedau, M.-Y. Im, P. Fischer, D. Arena, and A. Kent, J. Magn. Magn. Mater. 324, 3629 (2012).

${ }^{12}$ O. Hellwig, A. Berger, J. B. Kortright, and E. E. Fullerton, J. Magn. Magn. Mater. 319, 13 (2007).

${ }^{13}$ N. J. Harmon, F. Macià, F. Wang, M. Wohlgenannt, A. D. Kent, and M. E. Flatté, "Theory of organic magnetoresistance influenced by a ferromagnet's fringe field," Phys. Rev. B (submitted).

${ }^{14}$ Z. G. Yu, Phys. Rev. Lett. 106, 106602 (2011). 